\title{
A new construction of rational electromagnetic knots
}

Olaf Lechtenfeld*

Leibniz Universität Hannover, Germany

E-mail: olaf.lechtenfeldeitp.uni-hannover.de

\section{Gleb Zhilin}

Leibniz University Hannover, Germany

Belarusian State University, Minsk, Belarus

E-mail: gleb.zhilin@math.uni-hannover.de

\begin{abstract}
We set up a correspondence between solutions of the Yang-Mills equations on $\mathbb{R} \times S^{3}$ and in Minkowski spacetime via de Sitter space. Some known Abelian and non-Abelian exact solutions are rederived. For the Maxwell case we present a straightforward algorithm to generate an infinite number of explicit solutions, with fields and potentials in Minkowski coordinates given by rational functions of increasing complexity. We illustrate our method with some nontrivial examples.
\end{abstract}

Corfu Summer Institute 2018 "School and Workshops on Elementary Particle Physics and Gravity" (CORFU2018)

31 August - 28 September, 2018

Corfu, Greece

${ }^{*}$ Speaker. 


\section{Conformal equivalence of $\mathbf{d S}_{4}$ to $\mathscr{I} \times S^{3}$ and two copies of $\mathbb{R}_{+}^{1,3}$}

Four-dimensional de Sitter space is a one-sheeted hyperboloid (of radius $\ell$ ) in $\mathbb{R}^{1,4} \ni\left\{Z_{0}, Z_{1}, \ldots, Z_{4}\right\}$ given by

$$
-Z_{0}^{2}+Z_{1}^{2}+Z_{2}^{2}+Z_{3}^{2}+Z_{4}^{2}=\ell^{2}
$$

Constant $Z_{0}$ slices are 3 -spheres of varying radius, yielding a parametrization of $\mathrm{dS}_{4} \ni\left\{\tau, \omega_{A}\right\}$ as

$$
\begin{aligned}
& Z_{0}=-\ell \cot \tau \quad \text { and } \quad Z_{A}=\frac{\ell}{\sin \tau} \omega_{A} \quad \text { for } \quad A=1, \ldots, 4 \\
& \text { with } \quad \tau \in \mathscr{I}:=(0, \pi) \quad \text { and } \quad \omega_{A} \omega_{A}=1 .
\end{aligned}
$$

The Minkowski metric

$$
\mathrm{d} s^{2}=-\mathrm{d} Z_{0}^{2}+\mathrm{d} Z_{1}^{2}+\mathrm{d} Z_{2}^{2}+\mathrm{d} Z_{3}^{2}+\mathrm{d} Z_{4}^{2}
$$

induces on $\mathrm{dS}_{4}$ the metric

$$
\mathrm{d} s^{2}=\frac{\ell^{2}}{\sin ^{2} \tau}\left(-\mathrm{d} \tau^{2}+\mathrm{d} \Omega_{3}^{2}\right) \quad \text { with } \quad \mathrm{d} \Omega_{3}^{2} \text { for } S^{3},
$$

showing that $\mathrm{dS}_{4}$ is conformally equivalent to a finite cylinder $\mathscr{I} \times S^{3}$.

The $Z_{0}+Z_{4}<0$ half of $\mathrm{dS}_{4}$ is also conformally related to future Minkowski space $\mathbb{R}_{+}^{1,3} \ni$ $\{t, x, y, z\}$,

$$
\begin{aligned}
& Z_{0}=\frac{t^{2}-r^{2}-\ell^{2}}{2 t}, \quad Z_{1}=\ell \frac{x}{t}, \quad Z_{2}=\ell \frac{y}{t}, \quad Z_{3}=\ell \frac{z}{t}, \quad Z_{4}=\frac{r^{2}-t^{2}-\ell^{2}}{2 t} \\
& \text { with } \quad x, y, z \in \mathbb{R} \quad \text { and } \quad r^{2}=x^{2}+y^{2}+z^{2} \quad \text { but } \quad t \in \mathbb{R}_{+},
\end{aligned}
$$

since $t \in[0, \infty]$ corresponds to $Z_{0} \in[-\infty, \infty]$ but $Z_{0}+Z_{4}<0$. In these Minkowski coordinates,

$$
\mathrm{d} s^{2}=\frac{\ell^{2}}{t^{2}}\left(-\mathrm{d} t^{2}+\mathrm{d} x^{2}+\mathrm{d} y^{2}+\mathrm{d} z^{2}\right)
$$

One may cover the entire $\mathbb{R}^{1,3}$ by gluing a second $\mathrm{dS}_{4}$ copy and using the patch $Z_{0}+Z_{4}>0$.

We shall employ the direct relation between the cylinder and Minkowski coordinates:

$$
\cot \tau=\frac{r^{2}-t^{2}+\ell^{2}}{2 \ell t}, \quad \omega_{1}=\gamma \frac{x}{\ell}, \quad \omega_{2}=\gamma \frac{y}{\ell}, \quad \omega_{3}=\gamma \frac{z}{\ell}, \quad \omega_{4}=\gamma \frac{r^{2}-t^{2}-\ell^{2}}{2 \ell^{2}}
$$

with the convenient abbreviation

$$
\gamma=\frac{2 \ell^{2}}{\sqrt{4 \ell^{2} t^{2}+\left(r^{2}-t^{2}+\ell^{2}\right)^{2}}}
$$

Since $t=-\infty, 0, \infty$ corresponds to $\tau=-\pi, 0, \pi$, the cylinder gets doubled to $2 \mathscr{I} \times S^{3}$, and full Minkowski space is covered by the cylinder patch $\omega_{4} \leq \cos \tau$. The cylinder time $\tau$ is a regular smooth function of $(t, x, y, z)$, but more useful will be

$$
\exp (\mathrm{i} \tau)=\frac{(\ell+\mathrm{i} t)^{2}+r^{2}}{\sqrt{4 \ell^{2} t^{2}+\left(r^{2}-t^{2}+\ell^{2}\right)^{2}}} .
$$

The following is a rendition of the our publication [1]. 


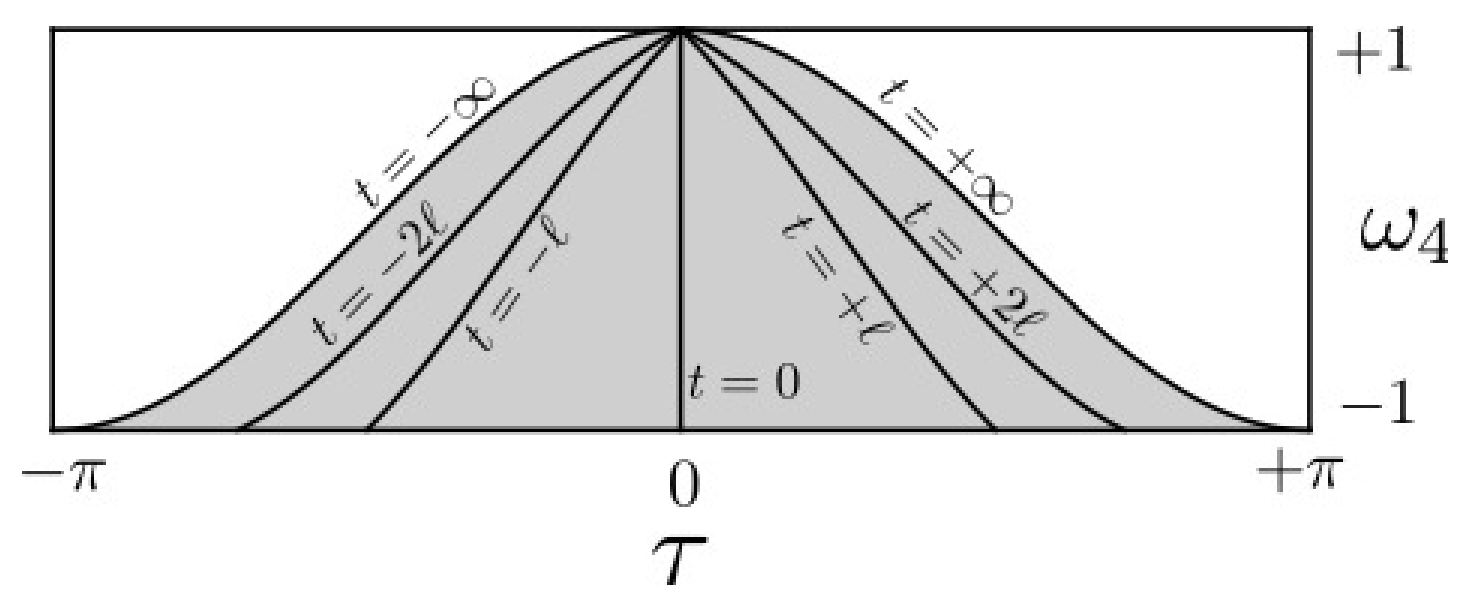

Figure 1: An illustration of the map between a cylinder $2 \mathscr{I} \times S^{3}$ and Minkowski space $\mathbb{R}^{1,3}$. The Minkowski coordinates cover the shaded area. Its boundary is given by the curve $\omega_{4}=\cos \tau$. Each point is a two-sphere spanned by $\omega_{1,2,3}$, which is mapped to a sphere of constant $r$ and $t$.

\section{The correspondence}

Yang-Mills and Maxwell theory are conformally invariant in four spacetime dimensions. Therefore, we may solve their equations of motion on the cylinder $2 \mathscr{I} \times S^{3}$ rather than directly on Minkowski space $\mathbb{R}^{1,3}$. The cylinder parametrization has the advantage that it makes manifest a hidden $\mathrm{SO}(4)$ covariance.

The gauge potential taking values in a Lie algebra $\mathfrak{g}$ can always be chosen as

$$
A=\sum_{a=1}^{3} X_{a}(\tau, \omega) e^{a} \quad \text { on } \quad 2 \mathscr{I} \times S^{3}
$$

where $X_{a} \in \mathfrak{g}$, and $\left\{e^{a}\right\}$ is a basis of left-invariant one-forms on $S^{3}$. There is no $\mathrm{d} \tau$ component because we picked the temporal gauge $A_{\tau}=0$.

Yang-Mills or Maxwell solutions are translated from $2 \mathscr{I} \times S^{3}$ to $\mathbb{R}^{1,3}$ simply by the coordinate change (1.7). The behavior at the boundary $\cos \tau=\omega_{4}$ yields the fall-off properties at $t \rightarrow \pm \infty$. To become explicit, we need the Minkowski-parametrization of the one-forms $e^{0} \equiv \mathrm{d} \tau$ and $e^{a}$, which are subject to

$$
\mathrm{d} e^{a}+\varepsilon_{b c}^{a} e^{b} \wedge e^{c}=0 \quad \text { and } \quad e^{a} e^{a}=\mathrm{d} \Omega_{3}^{2} .
$$

In terms of the $S^{3}$ coordinates $(a, i, j, k=1,2,3)$ they are

$$
e^{a}=-\eta_{B C}^{a} \omega_{B} \mathrm{~d} \omega_{C} \quad \text { where } \quad \eta_{j k}^{i}=\varepsilon_{j k}^{i} \eta_{j 4}^{i}=-\eta_{4 j}^{i}=\delta_{j}^{i} .
$$

A slightly lengthy computation yields the Minkowski-coordinate expressions,

$$
\begin{aligned}
& e^{0}=\frac{\gamma^{2}}{\ell^{3}}\left(\frac{1}{2}\left(t^{2}+r^{2}+\ell^{2}\right) \mathrm{d} t-t x^{k} \mathrm{~d} x^{k}\right) \\
& e^{a}=\frac{\gamma^{2}}{\ell^{3}}\left(t x^{a} \mathrm{~d} t-\left(\frac{1}{2}\left(t^{2}-r^{2}+\ell^{2}\right) \delta_{k}^{a}+x^{a} x^{k}+\ell \varepsilon_{j k}^{a} x^{j}\right) \mathrm{d} x^{k}\right),
\end{aligned}
$$


with the notation

$$
\left(x^{i}\right)=(x, y, z) \quad \text { and (for later) } \quad\left(x^{\mu}\right)=\left(x^{0}, x^{i}\right)=(t, x, y, z) .
$$

The simplest Yang-Mills solutions are most symmetric. To obtain them, let us impose $\mathrm{SO}(4)$ symmetry by setting $X_{a}(\tau, \omega)=X_{a}(\tau)$. The Yang-Mills equations then become ordinary matrix differential equations $[2,3]$,

$$
\frac{\mathrm{d}^{2}}{\mathrm{~d} \tau^{2}} X_{a}=-4 X_{a}+3 \varepsilon_{a b c}\left[X_{b}, X_{c}\right]-\left[X_{b},\left[X_{a}, X_{b}\right]\right] \quad\left[\frac{\mathrm{d}}{\mathrm{d} \tau} X_{a}, X_{a}\right]=0 .
$$

For $\mathfrak{g}=s u(2)$, these equations admit some analytic solutions $[4,5]$,

$$
X_{a}(\tau)=\left(1+\frac{1}{2} q(\tau)\right) T_{a} \quad \text { with } \quad \frac{\mathrm{d}^{2} q}{\mathrm{~d} \tau^{2}}=-\frac{\partial V}{\partial q} \quad \text { for } \quad V(q)=\frac{1}{2} q^{2}(q+2)^{2},
$$

where $\left\{T_{a}\right\}$ is an $\operatorname{su}(2)$ basis normalized to obey $\left[T_{a}, T_{b}\right]=2 \varepsilon_{a b c} T_{c}$. Notice the identification of Liealgebra and spatial indices. So the Yang-Mills problem has been reduced to a Newtonian particle in a double-well potential $V(q)$. Its prominent trajectories are (a) the vacua $q(\tau) \equiv-2$ or 0 , (b) the sphaleron $q(\tau) \equiv-1$ and (c) the bounce $q(\tau)=\sqrt{2} \operatorname{sech}\left(\sqrt{2}\left(\tau-\tau_{0}\right)\right)-1$. The corresponding gauge potential takes the form

$$
A=\left(1+\frac{1}{2} q(\tau)\right) g^{-1} \mathrm{~d} g \quad \text { for } \quad g: S^{3} \rightarrow \mathrm{SU}(2) .
$$

The sphaleron gives the only nontrivial static homogeneous solution (on the cylinder), i.e. $A=$ $\frac{1}{2} T_{a} e^{a}=\frac{1}{2} g^{-1} \mathrm{~d} g$, which translates to a finite-action homogeneous color-magnetic Yang-Mills solution on $\mathrm{dS}_{4}[6]$ (see also [7]).

In addition, there exist analytic Abelian symmetric solutions,

$$
X_{a}(\tau)=\bar{X}_{a}(\tau) T_{3} \quad \text { with } \quad \frac{\mathrm{d}^{2} \bar{X}_{a}}{\mathrm{~d} \tau^{2}}=-4 \bar{X}_{a} .
$$

Obviously, these are solutions to Maxwell's equations, taking $\mathfrak{g}=\mathbb{R}$, so we can drop the matrix $T_{3}$ and consider just real-valued functions $\bar{X}_{a}(\tau)$. Let us drop the bar and consider $X_{a} \in \mathbb{R}$ from now on. The general solution to (2.9) is an oscillation with frequency two,

$$
X_{a}(\tau)=c_{a} \cos \left(2\left(\tau-\tau_{a}\right)\right)
$$

The task is to transfer the oscillatory cylinder solutions to Minkowski space $\left(x \equiv\left\{x^{\mu}\right\}\right)$,

$$
\begin{array}{r}
A=X_{a}(\tau(x)) e^{a}(x)=A_{\mu}(x) \mathrm{d} x^{\mu} \quad \text { yielding } \quad A_{\mu}(x) \quad \text { with } \quad A_{t} \neq 0, \\
\mathrm{~d} A=\frac{\mathrm{d}}{\mathrm{d} \tau} X_{a} e^{0} \wedge e^{a}-\varepsilon_{b c}^{a} X_{a} e^{b} \wedge e^{c}=\frac{1}{2} F_{\mu \nu} \mathrm{d} x^{\mu} \wedge \mathrm{d} x^{v} \quad \text { yielding } \quad F_{\mu v}(x) .
\end{array}
$$

From this, we obtain electric and magnetic fields $E_{i}=F_{i 0} B_{i}=\frac{1}{2} \varepsilon_{i j k} F_{j k}$. For the computation it is helpful to recognize that $\exp (2 \mathrm{i} \tau)$ is a rational function of $t$ and $r$. 
We may always choose a frame where $X_{3}=0$ and $\tau_{2}=0$. The overall amplitude is irrelevant as all equations are linear, and solutions can be superposed at will. Specializing to $c_{1}=c_{2}=-\frac{1}{8}$ and $\tau_{1}=\frac{\pi}{2}$,

$$
X_{1}(\tau)=-\frac{1}{8} \sin 2 \tau, \quad X_{2}(\tau)=-\frac{1}{8} \cos 2 \tau, \quad X_{3}(\tau)=0,
$$

the result of short computation (putting $\ell=1$ ) yields

$$
\vec{E}+\mathrm{i} \vec{B}=\frac{1}{\left((t-\mathrm{i})^{2}-r^{2}\right)^{3}}\left(\begin{array}{c}
(x-\mathrm{i} y)^{2}-(t-\mathrm{i}-z)^{2} \\
\mathrm{i}(x-\mathrm{i} y)^{2}+\mathrm{i}(t-\mathrm{i}-z)^{2} \\
-2(x-\mathrm{i} y)(t-\mathrm{i}-z)
\end{array}\right) .
$$

This is the celebrated Hopf-Rañada electromagnetic knot [8, 9]. Our approach also yields its gauge potential.

\section{Construction of electromagnetic solutions}

In the following, we are interested only in Maxwell solutions. The linearity of the equations then will allow us to solve for a general (not $\mathrm{SO}(4)$-symmetric) potential. Therefore, let us admit arbitrary non-symmetric configurations $X_{a}=X_{a}(\tau, \omega)$ but capture the $\omega$-dependence in an $\mathrm{SO}(4)$-covariant fashion. The main ingredients are the left-invariant vector fields generating right multiplication,

$$
R_{a}=-\eta_{B C}^{a} \omega_{B} \frac{\partial}{\partial \omega_{C}} \quad \Rightarrow \quad\left[R_{a}, R_{b}\right]=2 \varepsilon_{a b c} R_{c}
$$

and the right-invariant ones generating left multiplication (by the inverse),

$$
L_{a}=-\tilde{\eta}_{B C}^{a} \omega_{B} \frac{\partial}{\partial \omega_{C}} \quad \Rightarrow \quad\left[L_{a}, L_{b}\right]=2 \varepsilon_{a b c} L_{c}
$$

They mutually commute, $\left[R_{a}, L_{b}\right]=0$, and the right translations are dual to our left-invariant oneforms, e.g. $e^{a}\left(R_{b}\right)=\delta_{b}^{a}$. Hence, an arbitrary function $\Phi$ on $S^{3}$ obeys

$$
\mathrm{d} \Phi(\omega)=e^{a} R_{a} \Phi(\omega) .
$$

The space of functions on $S^{3}$ decomposes into irreps of $s u(2)_{L} \oplus s u(2)_{R}$ labelled by a common spin $j \in\left\{0, \frac{1}{2}, 1, \frac{3}{2}, \ldots\right\}$. To make contact with standard physics notation, we define hermitian "angular momenta"

$$
I_{a}:=\frac{\mathrm{i}}{2} L_{a} J_{a}:=\frac{\mathrm{i}}{2} R_{a} \quad \Rightarrow \quad\left[I_{a}, I_{b}\right]=\mathrm{i} \varepsilon_{a b c} I_{c}\left[J_{a}, J_{b}\right]=\mathrm{i} \varepsilon_{a b c} J_{c} .
$$

A basis of hyperspherical harmonics

$$
Y_{j ; m, n}(\omega) \quad \text { with } \quad m, n=-j,-j+1, \ldots,+j \quad \text { and } \quad 2 j=0,1,2, \ldots
$$

is specified by the relations

$$
\begin{aligned}
& I^{2} Y_{j ; m, n}=J^{2} Y_{j ; m, n}=j(j+1) Y_{j ; m, n}, \\
& I_{3} Y_{j ; m, n}=m Y_{j ; m, n} \quad \text { and } \quad J_{3} Y_{j ; m, n}=n Y_{j ; m, n} .
\end{aligned}
$$


For an explicit construction, one introduces two complex coordinates

$$
\alpha=\omega_{1}+\mathrm{i} \omega_{2} \quad \text { and } \quad \beta=\omega_{3}+\mathrm{i} \omega_{4} \quad \text { subject to } \quad \bar{\alpha} \alpha+\bar{\beta} \beta=1 .
$$

The angular momenta generators in those terms read

$$
\begin{aligned}
I_{+} & =\left(\bar{\beta} \partial_{\bar{\alpha}}-\alpha \partial_{\beta}\right) / \sqrt{2}, & J_{+} & =\left(\beta \partial_{\bar{\alpha}}-\alpha \partial_{\bar{\beta}}\right) / \sqrt{2}, \\
I_{3} & =\left(\alpha \partial_{\alpha}+\bar{\beta} \partial_{\bar{\beta}}-\bar{\alpha} \partial_{\bar{\alpha}}-\beta \partial_{\beta}\right) / 2, & J_{3} & =\left(\alpha \partial_{\alpha}+\beta \partial_{\beta}-\bar{\alpha} \partial_{\bar{\alpha}}-\bar{\beta} \partial_{\bar{\beta}}\right) / 2, \\
I_{-} & =\left(\bar{\alpha} \partial_{\bar{\beta}}-\beta \partial_{\alpha}\right) / \sqrt{2}, & J_{-} & =\left(\bar{\alpha} \partial_{\beta}-\bar{\beta} \partial_{\alpha}\right) / \sqrt{2} .
\end{aligned}
$$

The normalized hyperspherical harmonics are represented as

$$
Y_{j ; m, n}=\sqrt{\frac{2 j+1}{2 \pi^{2}}} \sqrt{\frac{2^{j-m}(j+m) !}{(2 j) !(j-m) !} \frac{2^{j-n}(j+n) !}{(2 j) !(j-n) !}}\left(I_{-}\right)^{j-m}\left(J_{-}\right)^{j-n} \alpha^{2 j}
$$

and are homogenous polynomials of degree $2 j$ in $\{\alpha, \bar{\alpha}, \beta, \bar{\beta}\}$.

To set up a left-invariant and right-covariant formulation, we parametrize the general Maxwellian gauge potential on $2 \mathscr{I} \times S^{3}$ as

$$
A=X_{0}(\tau, \omega) \mathrm{d} \tau+X_{a}(\tau, \omega) e^{a}
$$

The temporal and Coulomb gauge allows us to impose

$$
X_{0}(\tau, \omega)=0 \quad \text { and } \quad J_{a} X_{a}(\tau, \omega)=0 .
$$

Maxwell's equations then are nothing but coupled wave equations:

$$
-\frac{1}{4} \partial_{\tau}^{2} X_{a}=\left(J^{2}+1\right) X_{a}+\mathrm{i} \varepsilon_{a b c} J_{b} X_{c}
$$

A more transparent rewriting employs the famiiar complex linear combinations

$$
X_{ \pm}=\left(X_{1} \pm \mathrm{i} X_{2}\right) / \sqrt{2}
$$

which provides a partial decoupling of the components,

$$
\begin{aligned}
& -\frac{1}{4} \partial_{\tau}^{2} X_{+}=\left(J^{2}-J_{3}+1\right) X_{+}+J_{+} X_{3}, \\
& -\frac{1}{4} \partial_{\tau}^{2} X_{3}=\left(J^{2}+1\right) X_{3}-J_{+} X_{-}+J_{-} X_{+}, \\
& -\frac{1}{4} \partial_{\tau}^{2} X_{-}=\left(J^{2}+J_{3}+1\right) X_{-}-J_{-} X_{3},
\end{aligned}
$$

to be supplemented by the gauge condition

$$
0=J_{3} X_{3}+J_{+} X_{-}+J_{-} X_{+}
$$

Since the $X_{a}$ live on $S^{3}$, we naturally expand in our basis of hyperspherical harmonics,

$$
X_{a}(\tau, \omega)=\sum_{j m n} X_{a}^{j ; m, n}(\tau) Y_{j ; m, n}(\alpha, \beta)
$$

From the form of the equations it is obvious that 
- the equations are diagonal in $j$ and $m$, so these may be kept fixed

- they only couple triplets $\left(X_{3}^{j ; m, n}, X_{+}^{j ; m, n+1}, X_{-}^{j ; m, n-1}\right)$, so $X_{ \pm} \propto J_{ \pm} X_{3}$ for $X_{3} \propto Y_{j ; m, n}$

- the ansatz $X_{a}^{j ; m, n}(\tau)=\mathrm{e}^{\mathrm{i} \Omega_{a}^{j ; n} \tau} c_{a}^{j ; n}$ gives a linear system for $\Omega_{a}^{j ; n}$ and $c_{a}^{j ; n}$

The frequencies turn out to be integral,

$$
\Omega_{a}^{j ; n}= \pm 2(j+1) \quad \text { or } \quad \pm 2 j
$$

which produces two types of basis solutions:

- type I : $\quad j \geq 0, \quad m=-j, \ldots,+j, \quad n=-j-1, \ldots, j+1, \quad \Omega^{j}= \pm 2(j+1)$,

$$
\begin{aligned}
& X_{+}=\sqrt{(j-n)(j-n+1) / 2} \mathrm{e}^{ \pm 2(j+1) \mathrm{i} \tau} Y_{j ; m, n+1}, \\
& X_{3}=\sqrt{(j+1)^{2}-n^{2}} \mathrm{e}^{ \pm 2(j+1) \mathrm{i} \tau} Y_{j ; m, n}, \\
& X_{-}=-\sqrt{(j+n)(j+n+1) / 2} \mathrm{e}^{ \pm 2(j+1) \mathrm{i} \tau} Y_{j ; m, n-1},
\end{aligned}
$$

- type II : $j \geq 1, \quad m=-j, \ldots,+j, \quad n=-j+1, \ldots, j-1, \quad \Omega^{j}= \pm 2 j$,

$$
\begin{aligned}
& X_{+}=-\sqrt{(j+n)(j+n+1) / 2} \mathrm{e}^{ \pm 2 j \mathrm{i} \tau} Y_{j ; m, n+1}, \\
& X_{3}=\sqrt{j^{2}-n^{2}} \mathrm{e}^{ \pm 2 j \mathrm{i} \tau} Y_{j ; m, n}, \\
& X_{-}=\sqrt{(j-n)(j-n+1) / 2} \mathrm{e}^{ \pm 2 j \mathrm{i} \tau} Y_{j ; m, n-1} .
\end{aligned}
$$

Of course, a generic solution is some linear combination of the above. Due to the linearity of the equations, the overall scale of a solution is arbitrary.

\section{Some properties of the solutions}

Each complex solution yields two real ones, real part and imaginary part. For fixed spin $j$ we get $2(2 j+1)(2 j+3)$ type-I solutions $(j \geq 0)$ and $2(2 j+1)(2 j-1)$ type-II solutions $(j>0)$. They add up to $4(2 j+1)^{2}$ solutions for $j>0$ and 6 solutions for $j=0$, which is the correct number for the dimension of a spin- $j$ representation of $\mathrm{SO}(4)$. Constant solutions $(\Omega=0)$ are not allowed; the simplest ones $(\Omega=2)$ are the three complex $j=0$ type I and three complex $j=1$ type II basis configurations $(j ; m, n)=(0, \star, 0)$ and $(1, \star, 0)$ with $\star=-1,0,+1$, respectively. The Hopf-Rañada solution is a real combination of $(0,+1,0)$ and $(0,-1,0)$. The classification (3.20) and (3.21) shows a general parity relation map between $(j ; m, n)$ type I and $(j+1 ; n, m)$ type II. Electromagnetic duality is realized via shifting $\left|\Omega^{j}\right| \tau$ by $\pm \frac{\pi}{2}$; this maps $A \mapsto A_{D}$.

The main technical task is to transform a chosen solution on $2 \mathscr{I} \times S^{3}$ to Minkowski coordinates $(t, x, y, z)$, which is straightforward due to the explicit formulæ for all ingredients and will produce only rational functions. Conserved (in time) quantities are helicity and energy,

$$
h=\frac{1}{2} \int_{\mathbb{R}^{3}}\left(A \wedge F+A_{D} \wedge F_{D}\right) \quad \text { and } \quad E=\frac{1}{2} \int_{\mathbb{R}^{3}} \mathrm{~d}^{3} x\left(\vec{E}^{2}+\vec{B}^{2}\right) .
$$


Their common scale is determined by the amplitude of the solution, but their ratio is fixed for the basis configurations. Both quantities are best computed in "sphere frame" at $t=\tau=0$,

$$
F=\mathscr{E}_{a} e^{a} \wedge e^{0}+\frac{1}{2} \mathscr{B}_{a} \varepsilon_{b c}^{a} e^{b} \wedge e^{c},
$$

giving, for example,

$$
\int_{\mathbb{R}^{3}} \mathrm{~d}^{3} x \vec{E}^{2}=\frac{1}{\ell} \int_{S^{3}} \mathrm{~d}^{3} \Omega_{3}\left(1-\omega_{4}\right) \mathscr{E}_{a} \mathscr{E}_{a} \quad \text { and } \quad \int_{\mathbb{R}^{3}} \mathrm{~d}^{3} x \vec{B}^{2}=\frac{1}{\ell} \int_{S^{3}} \mathrm{~d}^{3} \Omega_{3}\left(1-\omega_{4}\right) \mathscr{B}_{a} \mathscr{B}_{a},
$$

by exploiting the orthogonality properties of the hyperspherical harmonics.

\section{Examples}

Finally we shall present two cases for illustrative purposes. For the first example, let us take the real part of the $(j ; m, n)=(1 ; 0,0)$ type-I basis solution. Combining $\mathrm{e}^{4 \mathrm{i} \tau}+\mathrm{e}^{-4 \mathrm{i} \tau}=2 \cos 4 \tau$ and reading off $Y_{1 ; 0, \star}$ from (3.20), we have

$$
X_{+}=-\frac{\sqrt{3}}{\pi} \alpha \beta \cos 4 \tau, \quad X_{3}=\frac{\sqrt{6}}{\pi}(\beta \bar{\beta}-\alpha \bar{\alpha}) \cos 4 \tau, \quad X_{-}=-\frac{\sqrt{3}}{\pi} \bar{\alpha} \bar{\beta} \cos 4 \tau .
$$

This solution has $h=12$ and $E=48 / \ell$ and takes the explicit form

$$
\begin{aligned}
&(E+\mathrm{i} B)_{x}=\frac{-2 \mathrm{i}}{\left((t-\mathrm{i})^{2}-x^{2}-y^{2}-z^{2}\right)^{5}} \times \\
& \times\left\{2 y+3 \mathrm{i} t y-x z+2 t^{2} y+2 \mathrm{i} t x z-8 x^{2} y-8 y^{3}+4 y z^{2}\right. \\
&+4 \mathrm{i} t^{3} y-6 t^{2} x z-8 \mathrm{i} t x^{2} y-8 \mathrm{i} t y^{3}+4 \mathrm{i} t y z^{2}+10 x^{3} z+10 x y^{2} z-2 x z^{3} \\
&\left.+2\left(\mathrm{i} t x z+x^{2} y+y^{3}+y z^{2}\right)\left(-t^{2}+x^{2}+y^{2}+z^{2}\right)+(\mathrm{i} t y-x z)\left(-t^{2}+x^{2}+y^{2}+z^{2}\right)^{2}\right\}, \\
&(E+\mathrm{i} B)_{y}=\frac{2 \mathrm{i}}{\left((t-\mathrm{i})^{2}-x^{2}-y^{2}-z^{2}\right)^{5}} \times \\
& \times\left\{2 x+3 \mathrm{i} t x+y z+2 t^{2} x-2 \mathrm{i} t y z-8 x^{3}-8 x y^{2}+4 x z^{2}\right. \\
&+4 \mathrm{it} t^{3} x+6 t^{2} y z-8 \mathrm{i} t x^{3}-8 \mathrm{i} t x y^{2}+4 \mathrm{i} t x z^{2}-10 x^{2} y z-10 y^{3} z+2 y z^{3} \\
&+\left.2\left(-\mathrm{i} t y z+x^{3}+x y^{2}+x z^{2}\right)\left(-t^{2}+x^{2}+y^{2}+z^{2}\right)+(\mathrm{i} t x+y z)\left(-t^{2}+x^{2}+y^{2}+z^{2}\right)^{2}\right\}, \\
&(E+\mathrm{i} B)_{z}=\frac{\mathrm{i}}{\left((t-\mathrm{i})^{2}-x^{2}-y^{2}-z^{2}\right)^{5}} \times \\
& \times\left\{1+2 \mathrm{i} t+t^{2}-11 x^{2}-11 y^{2}+3 z^{2}+4 \mathrm{i} t^{3}-16 \mathrm{i} t x^{2}-16 \mathrm{i} t y^{2}+4 \mathrm{i} t z^{2}\right. \\
& \quad-t^{4}-2 t^{2} x^{2}-2 t^{2} y^{2}-2 t^{2} z^{2}+11 x^{4}+22 x^{2} y^{2}+10 x^{2} z^{2}+11 y^{4}-10 y^{2} z^{2}+3 z^{4} \\
&\left.+2 \mathrm{i} t\left(t^{2}-3 x^{2}-3 y^{2}-z^{2}\right)\left(t^{2}-x^{2}-y^{2}-z^{2}\right)-\left(t^{2}+x^{2}+y^{2}-z^{2}\right)\left(-t^{2}+x^{2}+y^{2}+z^{2}\right)^{2}\right\} .
\end{aligned}
$$

Figures 2 and 3 below show $t=0$ energy density level surfaces and a particular closed magnetic field line for this example. For the second example, a concrete $\left(\frac{3}{2} ; \frac{1}{2}, \frac{3}{2}\right)$ type-I solution, $t=0$ energy density level surfaces are displayed in Figure 4. 


\section{Summary and discussion}

- Rational electromagnetic fields with nontrivial topology have been investigated since 1989

- We introduced a new construction method based on two insights:

- the simplicity of solving Maxwell's equations on a temporal cylinder over a threesphere

- the conformal equivalence of a cylinder patch to four-dimensional Minkowski space

- $A=X_{v}(\tau, \omega) e^{v}=X_{v}(\tau(x), \omega(x)) e_{\mu}^{v}(x) \mathrm{d} x^{\mu}$

- Only finite-time $\tau \in(-\pi,+\pi)$ dynamics is required on the cylinder

- Our solutions have finite energy and action, by construction

- A complete basis was discovered for sufficiently fast spatially and temporally decaying fields

- The non-Abelian extension couples different $j$ components of $X_{a}$ and is expected to be much harder

- The method may be useful for a numerical study of Yang-Mills dynamics in Minkowski space

\section{Acknowledgments}

This work was partially supported by the Deutsche Forschungsgemeinschaft under grant LE 838/13 and by the Heisenberg-Landau program. It is based upon work from COST Action MP1405 QSPACE, supported by COST (European Cooperation in Science and Technology).

\section{References}

[1] O. Lechtenfeld and G. Zhilin, Phys. Lett. A 382 (2018) 1528 [arXiv: 1711.11144 [hep-th] ].

[2] T.A. Ivanova, O. Lechtenfeld and A.D. Popov, Phys. Rev. Lett. 119 (2017) 061601 [arXiv: 1704.07456 [hep-th] ].

[3] T.A. Ivanova and O. Lechtenfeld, Phys. Lett. B 670 (2008) 91 [arXiv: 0806.0394 [hep-th]].

[4] M. Lüscher, Phys. Lett. B 70 (1977) 321.

[5] A. Actor, Rev. Mod. Phys. 51 (1979) 461.

[6] V. de Alfaro, S. Fubini and G. Furlan, Phys. Lett. B 65 (1976) 163.

[7] G.W. Gibbons and A.R. Steif, Phys. Lett. B 346 (1995) 255.

[8] A.R. Rañada, Lett. Math. Phys. 18 (1989) 97.

[9] M. Arrayás, D. Bouwmeester and J.L. Trueba Phys. Rept. 667 (2017) 1. 


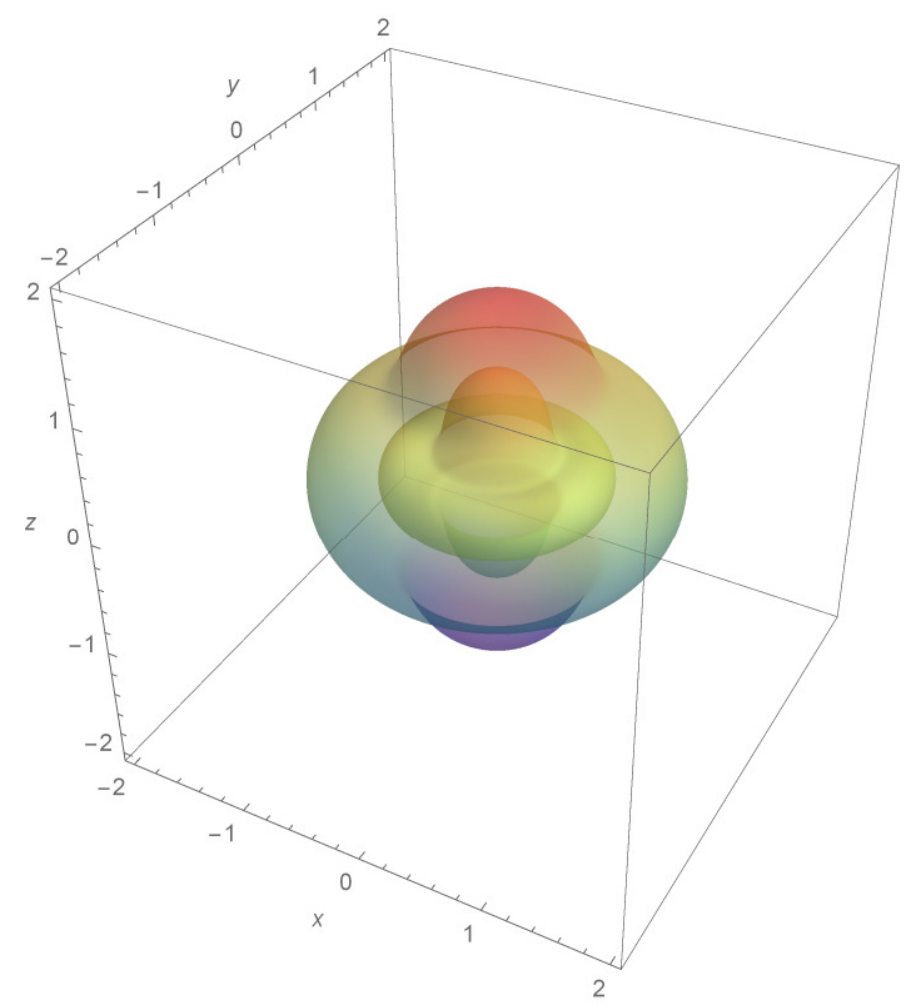

Figure 2: Energy density level surfaces at $t=0$ for the $(1 ; 0,0)$ solution above.

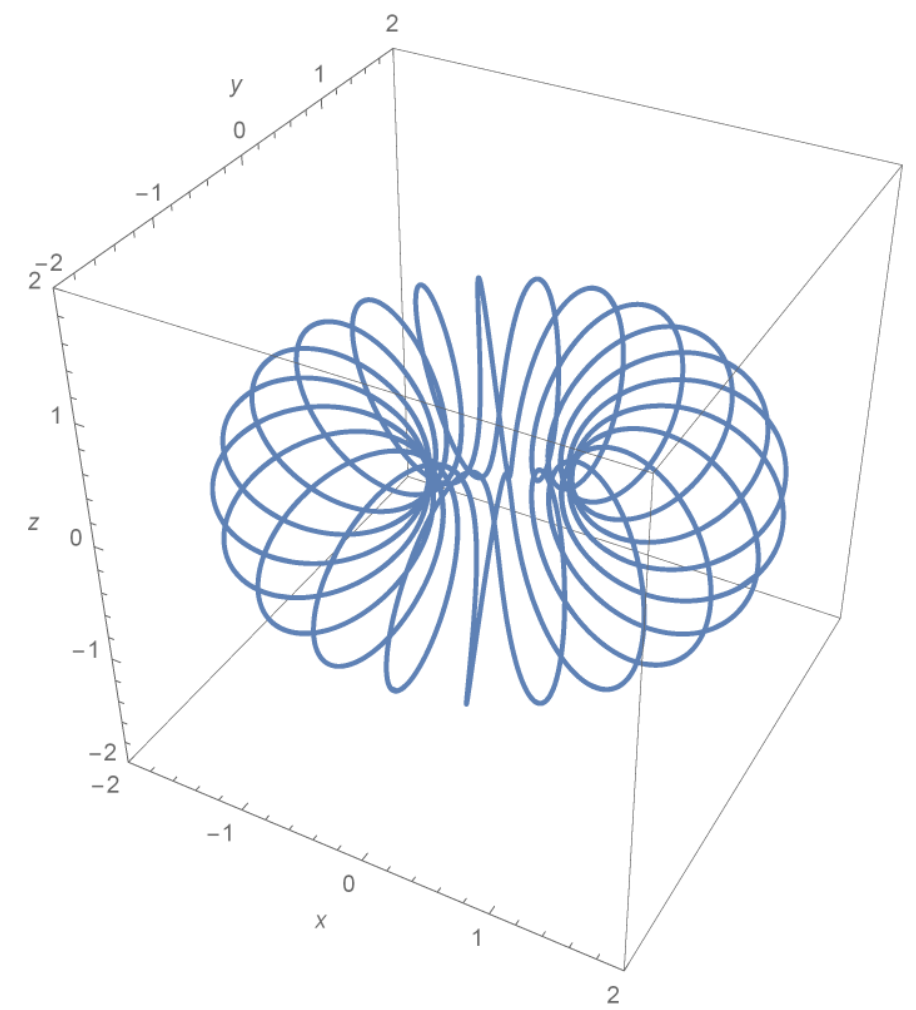

Figure 3: A particular magnetic field line for the $(1 ; 0,0)$ solution above. 


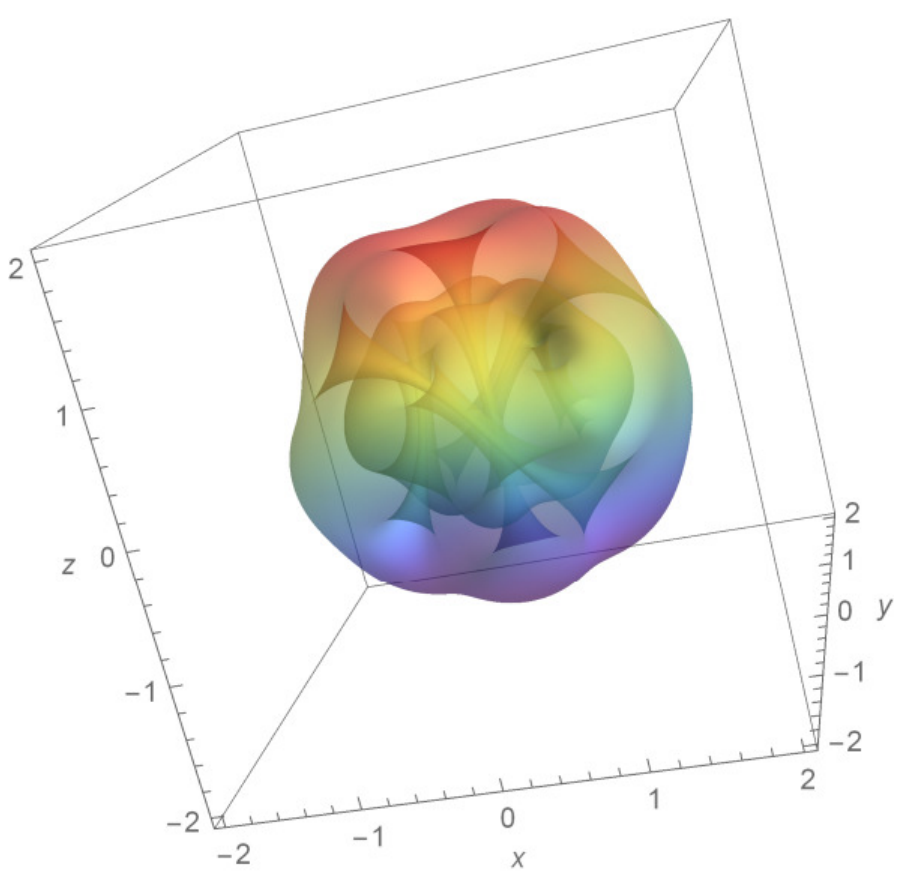

Figure 4: Energy density level surfaces at $t=0$ for a particular $\left(\frac{3}{2} ; \frac{1}{2}, \frac{3}{2}\right)$ solution. 